\title{
Estimation of blood velocity vectors using transverse ultrasound beam focusing and cross-correlation
}

Jensen, Jørgen Arendt; Lacasa, Isabel Rodriguez

Published in:

1999 IEEE Ultrasonics Symposium Proceedings

Link to article, DOI:

10.1109/ULTSYM.1999.849280

Publication date:

1999

Document Version

Publisher's PDF, also known as Version of record

Link back to DTU Orbit

Citation (APA):

Jensen, J. A., \& Lacasa, I. R. (1999). Estimation of blood velocity vectors using transverse ultrasound beam focusing and cross-correlation. In 1999 IEEE Ultrasonics Symposium Proceedings (Vol. 1-2, pp. 1493-1497). IEEE. I E E E International Ultrasonics Symposium. Proceedings https://doi.org/10.1109/ULTSYM.1999.849280

\section{General rights}

Copyright and moral rights for the publications made accessible in the public portal are retained by the authors and/or other copyright owners and it is a condition of accessing publications that users recognise and abide by the legal requirements associated with these rights.

- Users may download and print one copy of any publication from the public portal for the purpose of private study or research.

- You may not further distribute the material or use it for any profit-making activity or commercial gain

- You may freely distribute the URL identifying the publication in the public portal 


\title{
Estimation of blood velocity vectors using transverse ultrasound beam focusing and cross-correlation
}

\author{
Jørgen Arendt Jensen and Isabel Rodríguez Lacasa \\ Center for Fast Ultrasound Imaging, Department of Information Technology, Build. 344, \\ Technical University of Denmark, DK-2800 Lyngby, Denmark
}

\begin{abstract}
Modern ultrasound scanners estimate the blood velocity by tracking the movement of the blood scatterers along the ultrasound beam. This is done by emitting pulsed ultrasound fields and finding the shift in position from pulse to pulse by correlating the received signals. Only the velocity component along the beam direction is found, and this is a serious limitation in the current scanners, since most blood vessels are parallel to the skin surface. A method to find the velocity across the vessel has been suggested by Bonnefous [1]. Here a number of parallel receive beams are measured and used in a correlation estimator to find the velocity across the beam. This approach is extended in this paper by making beamforming along the direction of the flow. A fairly broad beam is emitted and the received signal is then focused along a selected direction. This direction can be along the ultrasound beam or across it or in any direction to the beam. The focused lines, thus, follow the flow and a cross-correlation of lines from different pulses can find the movement of the blood particles between pulse emissions and, thus, the blood velocity. The new approach is investigated using the Field II simulation program. Simulations are shown for a parabolic velocity profile for flow-to-beam angles of $30,45,60$, and 90 degrees using a 64 elements linear array with a center frequency of $3 \mathrm{MHz}$, a pitch of $0.3 \mathrm{~mm}$, and an element height of $5 \mathrm{~mm}$. The peak velocity in the parabolic flow was $0.5 \mathrm{~m} / \mathrm{s}$, and the pulse repetition frequency was $3.5 \mathrm{kHz}$. Using four pulse-echo lines, the parabolic flow profile was found with a standard deviation of $0.028 \mathrm{~m} / \mathrm{s}$ at 60 degrees and $0.092 \mathrm{~m} / \mathrm{s}$ at 90 degrees (transverse to the ultrasound beam), corresponding to accuracies of $5.6 \%$ and $18.4 \%$. Using ten lines gave standard deviations of $0.021 \mathrm{~m} / \mathrm{s}$ and $0.089 \mathrm{~m} / \mathrm{s}$, respectively, corresponding to accuracies of $4.2 \%$ and $17.8 \%$.
\end{abstract}

\section{Introduction}

Medical ultrasound systems are widely used for displaying a real time image of the blood flow in the human circulatory system. The velocity is estimated by finding the shift in position of the blood scatterers between pulse emissions along the direction of the ultrasound beam [2]. This can be done by either finding the phase shift between lines or by crosscorrelating the received RF signals and then find the position of the peak in the cross-correlation function. This directly gives the displacement and thereby the velocity. Only the displacement along the ultrasound beam is found, and this is a major limitation of current systems, since the blood flow is often parallel to the skin and thereby perpendicular to the ultrasound beam.

Several approaches have attempted to remedy this deficiency. Among these are speckle tracking [3], crossed beams [4], and transverse modulation beams [5], [6]. Bonnefous [1] suggested using a number of parallel beamformers to generate a signal transverse to the ultrasound beam from which the transverse velocity could be found. A single example for an entirely transverse flow was shown in [1], but no examples for other angles were given.

This paper extends Bonnefous' idea of lateral beamforming to perform the beamforming at a number of positions along a line in the direction of the flow vector. This makes it possible to find the flow at all angles and not only along or across the ultrasound beam. A beam focused orthogonal to the ultrasound beam will be influenced by the distribution of velocities as illustrated in Fig. 1. The beams will give a signal integrated over the velocity profile, and no unique velocity can be determined by the cross correlation estimator. Tilting the beams to follow the flow as in Fig. 2 makes it possible to uniquely identify the flow velocity. This is the approach taken in this paper. The flow angle is currently used in the approach and it must be set manually by the user as in traditional scanning.

\section{Theory}

The generation of the lateral focusing line requires a broad beam emission and multiple foci lines in reception. This can be achieved with a multi-element transducer. The focusing 


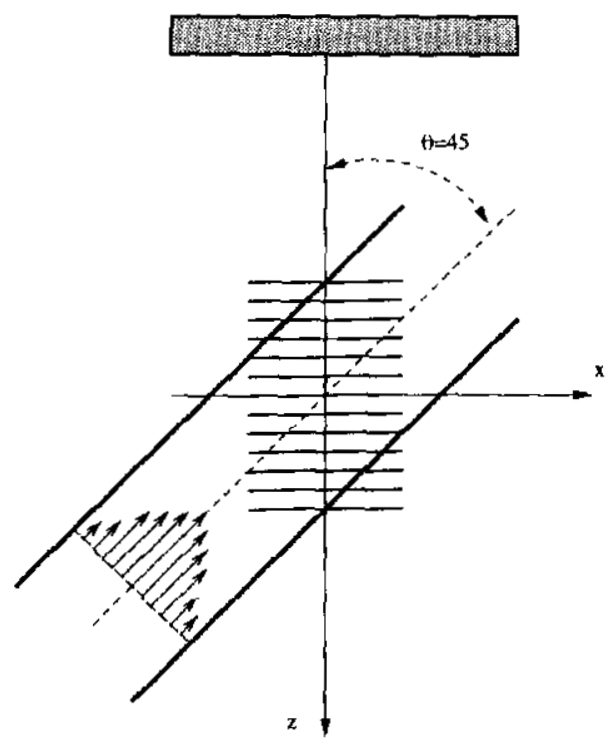

Figure 1: Transverse ultrasound beams used for estimating a parabolic flow profile.

line is situated over the region of interest, where the motion of the scatterers occurs, see Fig. 2. Along the whole length of the line the scatterers, which motion is tracked, must have the same velocity. For a laminar flow, the line, thus, has to be tilted in the direction of the flow lines.

The lateral focusing line is calculated during reception by delaying and adding responses from the individual elements of the array. The delays are found for each focus point along the line by determining the time it takes for the ultrasound wave to travel from each of the transducer elements to the focus point and back to the transducer. Lateral beams are in this way constructed for each of the transmitted ultrasound pulses.

The velocity is estimated from the cross-correlation of two consecutive focusing lines. In this case the displacement of the signals corresponds to an estimate of the distance traveled by the scatterers in the lateral beam direction. Since the lateral beams are situated along the flow stream-lines, the complete velocity vector is directly estimated.

\subsection{Measurement principle}

This section derives a mathematical model of the lateral beam approach. The measurement situation is depicted in Fig. 3. The figure shows a multi-element transducer with $N$ elements. The vector from the origin of reference to the physical center of element $i$ is $\vec{r}_{i}$. A tilted focus line, that follows the motion of the scatterers, is placed in the middle of the vessel. The vector $\vec{r}_{j}$ indicates the position of one of the $j$ focus points in the line, so that

$$
\vec{r}_{i}=\left(x_{i}, y_{i}, z_{i}\right) \quad i=1 \ldots N
$$

\section{4 - 1999 IEEE ULTRASONICS SYMPOSIUM}

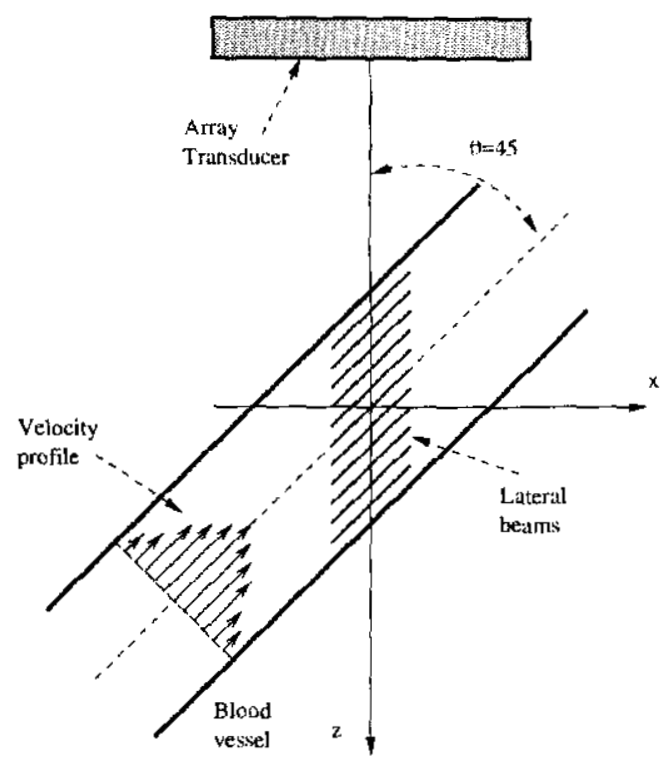

Figure 2: Focusing lines to obtain the lateral focusing lines for a parabolic velocity profile

$$
\begin{aligned}
\vec{r}_{j} & =\left(x_{j}, y_{j}, z_{j}\right) \quad j=1 \ldots M \\
\vec{r}_{i j} & =\vec{r}_{j}-\vec{r}_{i}=\left(x_{j}-x_{i}, y_{j}-y_{i}, z_{j}-z_{i}\right),
\end{aligned}
$$

where $\vec{r}_{i j}$ is the vector from element $i$ to the focus point $j$, and $M$ is the number of focus points in the line.

The time it takes the ultrasound pulse to propagate from element $i$ to the focus point $j$ and back to element $i$ is

$$
t_{i j}=\frac{2}{c}\left|\vec{r}_{i j}\right|=\frac{2}{c} \sqrt{\left(x_{j}-x_{i}\right)^{2}+\left(y_{j}-y_{i}\right)^{2}+\left(z_{j}-z_{i}\right)^{2}},
$$

when no delays are employed during emission of the ultrasound field. The received backscattered signal from element $i$ that corresponds to the ultrasound pulse emitted at $n T_{p r f}$, can be written as $r_{n}\left(\vec{r}_{i}, t\right)$, where $t$ indicates the time since pulse emission. The value of the field in a focus point $j$, is calculated by adding the responses found in each element $i$ for the appropriate delay time $t_{i j}$

$$
s_{n}\left(\vec{r}_{j}\right)=\sum_{i=1}^{N} r_{n}\left(\vec{r}_{i}, t_{i j}\right)
$$

where $N$ is the number of transducer elements. For the next ultrasound pulse $(n+1) T_{p r f}$, the value of the field in the same point will be

$$
\begin{aligned}
s_{n+1}\left(\vec{r}_{j}\right) & =\sum_{i=1}^{N} r_{n+1}\left(\vec{r}_{i}, t_{i j}\right) \\
& =\sum_{i=1}^{N} r_{n}\left(\vec{r}_{i}, t_{i j}-2 \frac{\nu_{i, a x i s}}{c} T_{p r}\right),
\end{aligned}
$$

where $v_{i, a x i s}$ represents the component of the velocity of a scatterer placed in $\vec{r}_{j}$, projected on the $\vec{r}_{i j}$ direction. The signal is, thus, delayed due to the motion of the scatterer between 


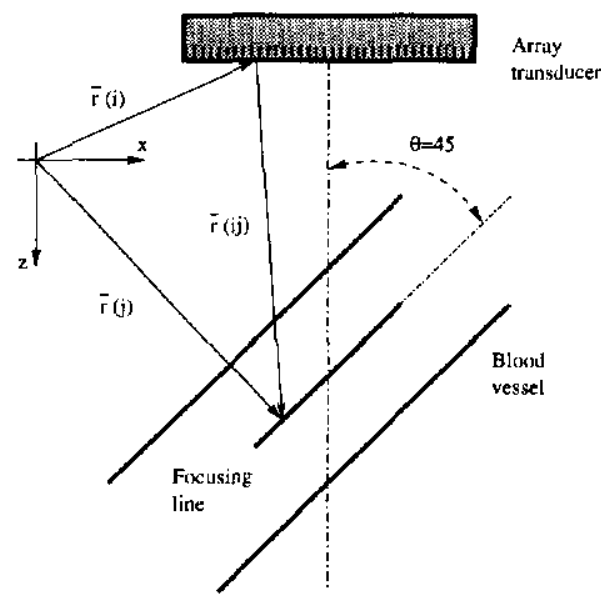

Figure 3: Coordinate system for the lateral focusing line measurement principle

pulse emissions. The time $t_{i j}-2 \frac{v_{i, a x i s}}{c} T_{p r f}$ will correspond to a delay value $t_{i k}$, where $k$ is another point on the focusing line. If $\vec{d}_{l}$ is a unitary vector in the direction of the lateral focusing line, and $l_{s}$ is the distance between the points $j$ and $k$, then $\vec{r}_{j}=\vec{r}_{k}+l_{s} \vec{d}_{l}$, and the following relation is found

$$
\begin{aligned}
& s_{n+1}\left(\vec{r}_{j}\right)=\sum_{i=1}^{N} r_{n}\left(\vec{r}_{i}, t_{i j}-2 \frac{v_{i, a x i s}}{c} T_{p r j}\right)=\sum_{i=1}^{N} r_{n}\left(\vec{r}_{i}, t_{i k}\right) \\
& s_{n+1}\left(\vec{r}_{j}\right)=s_{n}\left(\vec{r}_{k}\right)=s_{n}\left(\vec{r}_{j}-l_{s} \vec{d}_{l}\right)
\end{aligned}
$$

The focusing line signal calculated for the pulse emission at time $(n+1) T_{p r f}$ is, thus, a shifted version of the focusing line signal for pulse emission at time $n T_{p r f}$. Introducing a discrete version of the transverse signal gives

$$
S_{n}(j)=s_{n}\left(\vec{r}_{j}\right)
$$

The shift in lateral position can then be calculated from the cross-correlation of two consecutive signals $S_{1}[j]$ and $S_{2}[j]$ :

$$
\begin{aligned}
R_{12}(n) & =\frac{1}{M} \sum_{j=0}^{M-1} S_{1}[j] S_{2}[j+n] \\
& =\frac{1}{M} \sum_{j=0}^{M-1} S_{1} l[j] S_{1}\left[j+n-n_{s}\right] \\
R_{12}(n) & =R_{11}\left(n-n_{s}\right),
\end{aligned}
$$

where $R_{1 \mathrm{I}}(n)$ is the autocorrelation function of $S_{1}[j]$. The shift in position is then determined by the index of the peak of the cross-correlation function

$$
\operatorname{Max}_{n}\left[R_{12}(n)\right]=\operatorname{Max}_{n}\left[R_{11}\left(n-n_{s}\right)\right] \quad \Rightarrow \quad \hat{l}_{s}=\Delta l \hat{n}_{s}
$$

where $\Delta l$ is the lateral sampling interval. The crosscorrelation can be improved by averaging over several esti-

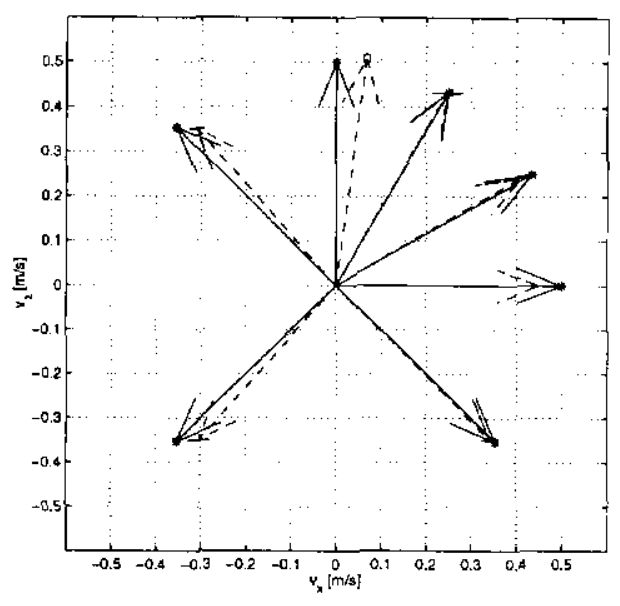

Figure 4: The continuous line arrows show the real velocity and the dashed line arrows show the estimated velocity: $\vec{v}_{e: t}=$ $\left(\hat{v}_{x}, \hat{v}_{z}\right)$.

mates of $R_{12}$, since the velocity of the scatterers can be considered constant for several pulses. The estimated velocity between two consecutive pulses is:

$$
\hat{v}=\frac{\Delta l}{T_{p r f}} \hat{n}_{s} .
$$

More information about the implementation of the approach can be found in [7].

\section{Results}

The new estimation approach was investigated using the Field II simulation program [8]. A $3 \mathrm{MHz} 64$ elements linear array transducer was used with a transmit focus at $150 \mathrm{~mm}$. The height of the elements was $5 \mathrm{~mm}$ and the pitch is $0.3 \mathrm{~mm}$. A two cycle pulse was used and the pulse repetition frequency was $3.5 \mathrm{kHz}$. A plug flow with a velocity equal to $v=0.5 \mathrm{~m} / \mathrm{s}$ and 5000 point scatterers was simulated. The center of the vessel was placed at a depth of $30 \mathrm{~mm}$ and the diameter of the vessel was $10 \mathrm{~mm}$.

The beamforming was here done orthogonal to the ultrasound beam, so that no tilting of the focusing line was performed. The axial and transverse velocities were both estimated using the cross-correlation approach. The axial time shift was estimated first and then used for off-setting the data for the orthogonal beamforming, so the axial motion was eliminated by compensating for the axial time shift.

Figure 4 shows the result of the simulations. The scatterer patterns were generated for seven different angles between the vessel and the axial beam. The received signals were found for 20 shoots. The cross-correlation estimator used 4 consecutive lines and this gave a total of 16 estimates for each velocity value. The dashed arrows point to 


\begin{tabular}{|l||c|c|c|c|c|c|}
\hline \multicolumn{1}{|c|}{$[\mathrm{deg}]$} & 0 & 30 & 60 & 90 & 135 & -135 \\
\hline \hline True $v_{x}[\mathrm{~m} / \mathrm{s}]$ & 0.0 & 0.25 & 0.43 & 0.50 & 0.35 & -0.35 \\
Mean $\hat{v}_{x}[\mathrm{~m} / \mathrm{s}]$ & 0.067 & 0.245 & 0.407 & 0.449 & 0.342 & -0.303 \\
Std $\hat{v}_{x}[\mathrm{~m} / \mathrm{s}]$ & 0.008 & 0.033 & 0.014 & 0.019 & 0.018 & 0.023 \\
Error $[\%]$ & - & -2.0 & -5.3 & -10.2 & 2.2 & 13.4 \\
Accuracy $[\%]$ & 11.9 & 13.4 & 3.4 & 4.2 & 5.2 & 7.5 \\
\hline
\end{tabular}

Table 1: Values of the correct transverse velocity and mean value and standard deviation of the estimates for plug flow. The error is calculated as: $\left(\right.$ mean $\left.\left(\hat{v}_{x}\right)-v_{x}\right) / v_{x}$, and the accuracy is mean $\left(\hat{v}_{x}\right) / \operatorname{std}\left(\hat{v}_{x}\right)$.

$\left(\operatorname{mean}\left(\hat{v}_{x}\right)\right.$, mean $\left.\left(\hat{v}_{z}\right)\right)$ for each angle, while the true values are represented by the solid line arrows. The standard deviation is shown for each estimated point as the axis of the following ellipse:

$$
\left(\frac{\operatorname{mean}\left(\hat{v}_{x}\right)}{\operatorname{std}\left(\hat{v}_{x}\right)}\right)^{2}+\left(\frac{\operatorname{mean}\left(\hat{v}_{z}\right)}{\operatorname{std}\left(\hat{v}_{z}\right)}\right)^{2}=1
$$

The estimates proved to be very close to the real velocities (see Table 1 ). The values for the axial velocity $\hat{v}_{z}$ were very close to the true velocity and the maximum standard deviation for $\hat{v}_{z}$, found for $\theta=30^{\circ}$, had a value of $0.0015 \mathrm{~m} / \mathrm{s}$.

\subsection{Results for a parabolic profile}

The next step was to simulate a parabolic profile for the scatterers and estimate the map of velocities with the lateral beam approach. The transducer and simulation setup was the same as for the plug flow.

Again orthogonal focusing lines, perpendicular to the $z$ axis, were calculated for different depths with a compensation for the axial velocity. No satisfactory results were obtained with this method. The second approach tilted the focused lines in the same direction as the scatterers motion (flow beams). Each line will track scatterers with the same velocity; the estimation of the velocity this approach gave satisfactory results.

The focusing lines for the flow beams were selected to be $10 \mathrm{~mm}$ long. They are rotated to have the same direction as the flow lines and are calculated for the different depths. The sampling interval of the axial position was $0.1 \mathrm{~mm}$ and the lateral sampling interval $\Delta l$ was $0.01 \mathrm{~mm}$.

Once the cross-correlation function is calculated and averaged over a number of focusing lines, the index of the maximum is obtained and interpolated to increase the velocity resolution. The interpolation was done by fitting a parabola to the cross-correlation function around the maximum [9]. The velocity is calculated by

$$
v=\frac{\Delta l}{T_{p r f}} \cdot \hat{n}_{i n t}
$$

where $n_{i n t}$ is the interpolated index.
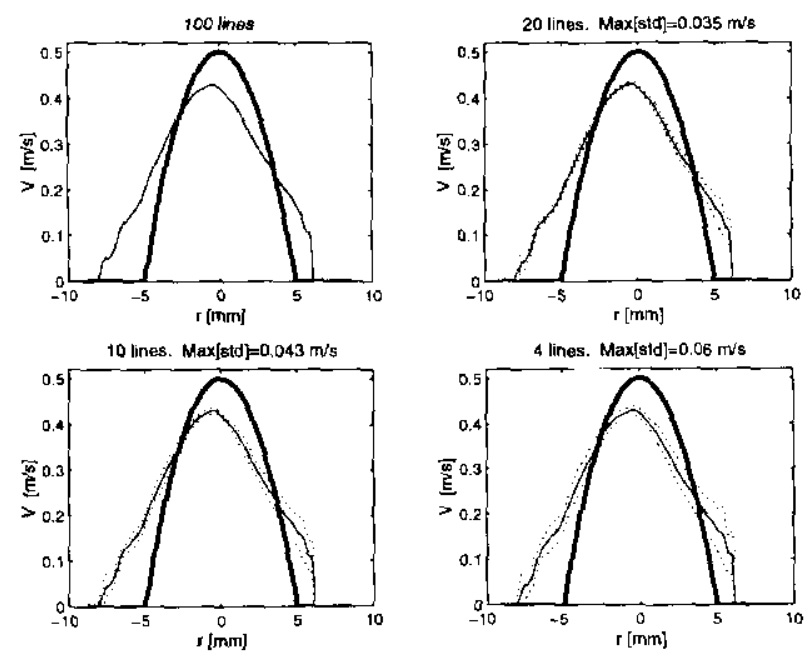

Figure 5: Estimated velocity profiles for $\theta=30^{\circ}$ using 100 , 20,10 and 4 lines. The thick line indicates the true velocity profile, the continuous line is the mean of the estimates, and the dotted lines are the mean \pm one standard deviation. The

\begin{tabular}{|c|c|c|c|c|}
\hline$\overline{\theta[\mathrm{deg}]}$ & 30 & $\overline{45}$ & 60 & 90 \\
\hline$\overline{\mathrm{Me}}$ & 081 & $0 . \overline{056}$ & $\overline{0.04}$ & .07 \\
\hline Std $\hat{i}_{\hat{i}}$ & 0.04 & 0.038 & 0.029 & 0.02 \\
\hline
\end{tabular}
maximum standard deviation is shown on top of each plot.

Table 2: Mean and standard deviation of the difference between the true profile and the estimated one.

The simulation was repeated for four inclinations of the vessel, $\theta=30,45,60$, and $90^{\circ}$. For each angle, the estimates were found by averaging over $4,10,20$ or 100 lines in the cross-correlation, which gave a total of $96,90,80$ or 1 velocity estimates respectively for every lateral beam. Some of the results are plotted in Figures 5, 6, and 7. The thick solid line is the true velocity profile, the thin solid line is the mean, and the dotted lines are the mean \pm one standard deviation of the estimates.

The total error in the velocity estimation is given by the mean and the standard deviation of the difference between the true and the estimated velocity profile as shown in Table 2. The values are for the estimates using 4 lines. The results are calculated for a different cross-section distance for each of the angles, so that only the significant part of the plots are taken into account : $r \in[-8.5,6]$ for $\theta=30^{\circ}, r \in[-8.3,8.3]$ for $\theta=45^{\circ}, r \in[-8,8]$ for $\theta=60^{\circ}, r \in[-5,5]$ for $\theta=90^{\circ}(r$ is the distance from the center of the vessel in $\mathrm{mm}$ ). 

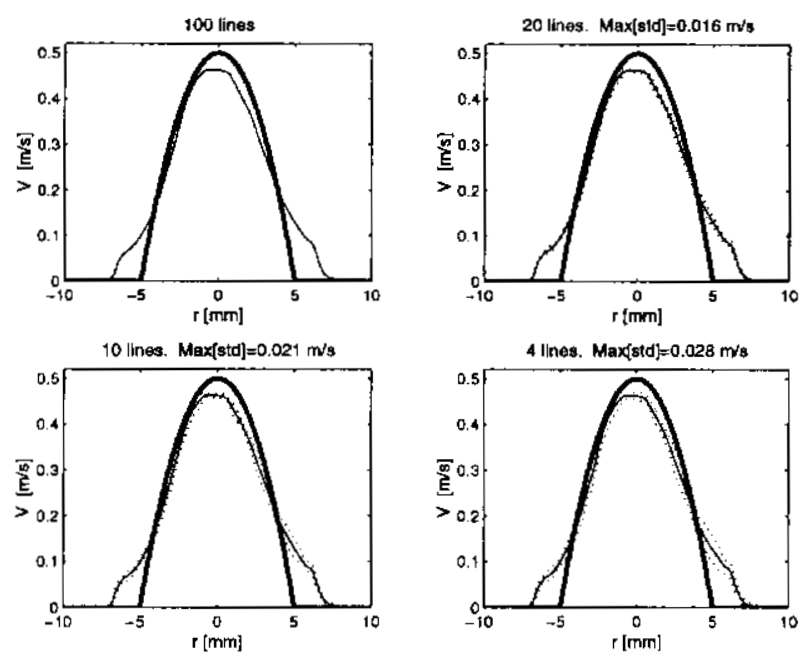

Figure 6: Estimated velocity profiles for $\theta=60^{\circ}$ using 100,20 , 10 and 4 lines.

\section{Summary of results}

The simulations for a parabolic flow shows that the new method can accurately estimate the velocity profile within a relative accuracy of $10 \%$ when using only four pulses emissions. The profile for angles different from $90^{\circ}$ is wider than the actual vessel due to the weakly focused transmit beam employed. The estimates are also biased around $30^{\circ}$ and the peak value in the vessel is underestimated. The underestimation is reduced for larger angles. A noisier estimate is found for an angle of $90^{\circ}$ than at other angles. The reason for this is yet unclear.

The angle of the flow must be entered into the estimator in order to generate the flow beams, and this is a major drawback of the approach. It can, however, probably be estimated from the actual data by also finding the peak in the crosscorrelation as a function of angle at the expense of an increase in the number of calculations. Another improvement is to use synthetic aperture imaging to increase the focusing of the transverse beams and thereby lower the widening of the estimated profiles.

\section{Acknowledgment}

This work was supported by grant 9700883 and 9700563 from the Danish Science Foundation and by B-K Medical $\mathrm{A} / \mathrm{S}$.

\section{References}

[1] O. Bonnefous. Measurement of the complete (3D) velocity vector of blood flows. In Proc. IEEE Ultrason. Symp.,
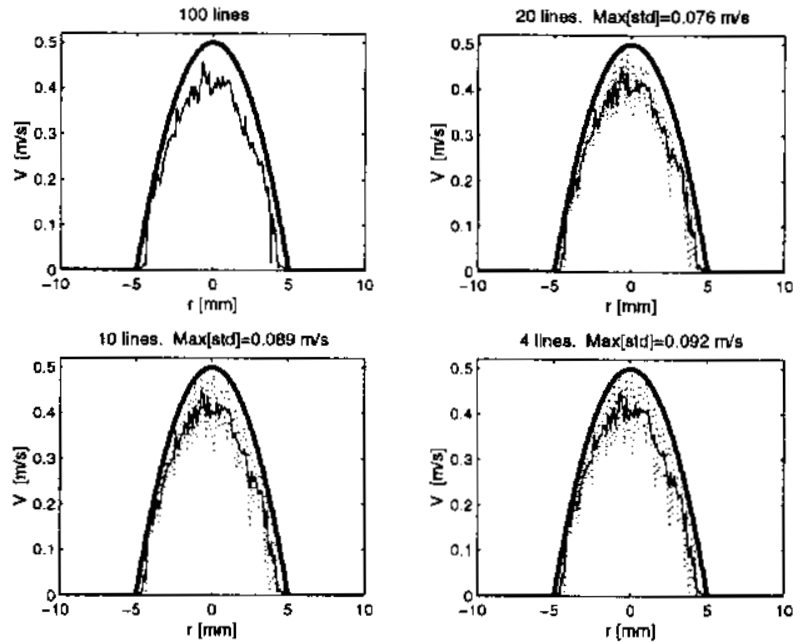

Figure 7: Estimated velocity profiles for $\theta=90^{\circ}$ using 100,20 , 10 and 4 lines.

pages $795-799,1988$.

[2] J. A. Jensen. Estimation of Blood Velocities Using Ultrasound: A Signal Processing Approach. Cambridge University Press, New York, 1996.

[3] G. E. Trahey, J. W. Allison, and O. T. von Ramm. Angle independent ultrasonic detection of blood flow. IEEE Trans. Biomed. Eng., BME-34:965-967, 1987.

[4] M. D. Fox. Multiple crossed-beam ultrasound Doppler velocimetry. IEEE Trans. Son. Ultrason., SU-25:281286,1978 .

[5] J. A. Jensen and P. Munk. A new method for estimation of velocity vectors. IEEE Trans. Ultrason., Ferroelec., Freq. Contr. 45:837-851, 1998.

[6] M. E. Anderson. Spatial quadrature: a novel technique for multi-dimensional velocity estimation. IEEE Trans. Ultrason., Ferroelec., Freq. Contr., 45:852-681, 1998.

[7] I. R. Lacasa. Estimation of blood velocities with ultrasound. Master's thesis, Department of Information Technology, Technical University of Denmark, 1999.

[8] J. A. Jensen. Field: A program for simulating ultrasound systems. Med. Biol. Eng. Comp., 10th Nordic-Baltic Conference on Biomedical Imaging, Vol. 4, Supplement 1, Part 1:351-353, 1996b.

[9] S. G. Foster. A pulsed ultrasonic flowmeter employing time domain methods. PhD thesis, Dept. Elec. Eng., University of Illinois, Urbana, Ill., 1985.

1999 IEEE ULTRASONICS SYMPOSIUM - 1497 